\section{FDA Recalls Blood Products After Donor Diagnosed with Creutzfeldt- Jakob Disease}

The FDA recently reported a voluntary market withdrawal of certain blood and plasma products by the American Red Cross, in cooperation with Baxter Healthcare Corporation and Miles Inc. The market withdrawal was initiated because a frequent Red Cross blood donor was diagnosed with Creutzfeldt-Jakob Disease (CJD).

Although there never has been a reported case of transmission of CJD by blood or plasma products, it is not known whether CJD can be transmitted by blood. However, FDA agreed with the Red Cross and Miles Inc. that it was prudent to withdraw the products to ensure the continued safety of the blood supply.

CJD is a fatal degenerative disease of the central nervous system thought be to caused by a virus-like agent. The annual incidence of CJD in the United States is less than one case per one million people. Patients harbor the agent for many years before developing symptoms. CJD has been transmitted to individuals who received certain human tissue transplant material, including corneas and brainassociated membrane, as well as patients treated with human pituitary-derived growth hormone. Blood from patients with CJD has been reported to transmit infection to animals if inoculated directly into the animal's brain.

FDA has consulted with the Centers for Disease Control and Prevention and the National Institute of Health to gather more information on the potential for transmission of CJD through transfusion and to assess the public health risk from various blood products when there is a CJD donor. The FDA will report the measures taken to its Blood Products Advisory Committee, a group of outside experts, for the purpose of reevaluating the current approach to product withdrawal.

FROM: FDA Talk Paper; November 17, 1994:T94-55.

\section{Elimination of Gowning in Neonatal Intensive Care Does Not Affect Infection Rates or Hand- Washing Patterns-Saves $\$ 120,000$ Annually}

The effect of gowning on colonization, infection, and hand-washing patterns in a neonatal intensive care unit (NICU) recently was studied by Susan Pelke and her colleagues at the Kapiolani Medical Center in Honolulu. Alternate Z-month gowning and no-gowning cycles were established in a 24-bed, level-III NICU for 8 months, with respiratory site, umbilical, and stool surveillance cultures performed weekly. Throughout the 8-month study period, an initial Z-minute hand scrub was recommended for staff and visitors, with a minimum of a 15 -second hand wash between handling infants. Without the knowledge of the staff, a neonatal research nurse observed hand-washing patterns.

During the gowning period, nursing staff, ward clerks, and residents changed into hospital-laundered scrub dresses or suits when they reported on duty. Long-sleeved cloth gowns were worn over the scrubs when they left the NICU. Other physicians, hospital staff, and visitors wore gowns over street clothes for entry into the NICU.

During the no-gowning period, nursing staff wore hospital-issued pant suits, washed at home through ordinary methods and worn from home. No gowns, jackets, or laboratory coats were worn outside the NICU. Ward clerks, physicians, hospital staff, families, and visitors wore street clothes without gowns. Only residents continued to wear hospital-laundered scrubs.

The results indicated that there was no significant difference between the gowning and no-gowning periods in the rate of any type of infection, including respiratory syncytial virus and necrotizing enterocolitis. There also was no difference in rates of bacterial colonization or any effect on hand-washing compliance.

A number of other studies in the NICU that have similar results have compared infection rates during periods of gowning as usual with periods of gowning only when infants are handled (modified gowning). This study is the largest of only two studies in the NICU in which gowning was compared to a period of no-gowning. Elimination of gowns saved this hospital more than $\$ 120,000$ on laundry and linen costs in one year.

FROM: Pelke S, Ching D, Easa D, Melish E. Gowning does not affect colonization or infection rates in a neonatal intensive care unit. Arch Pediatr Adolesc M ed 1994;148:10161020.

\section{Formula Supplemented with Bifidobacterium bifidum and Streptococcus thermophilus Prevents Diarrhea and Shedding of Rotavirus in Infants}

Acute diarrhea is a serious cause of infant morbidity and mortality. Bifidobacteria (which constitute the predominant intestinal flora of breast-fed infants), as well as other lactic acid-producing organisms, such as Streptococcus thermophilus, are thought to have a protective effect against acute diarrheal disease. However, their efficacy has not been assessed in controlled trials.

Dr. Jose Saavedra and colleagues from Johns Hopkins University School of Medicine and Mount Washington Pediatric Hospital in Baltimore recently reported the results of a study that found formula supplemented with $B$ bifidum and $S$ thermophilus reduced episodes of diarrhea and the shedding of rotavirus in infants.

In a double-blind placebo-controlled trial, infants aged 5 to 24 months who were admitted to a chronic medical care hospital were randomized to receive a standard infant formula or the same formula supplemented with $B$ bifidum and $S$ thermophilus. Eight $(26 \%)$ of the 26 patients who received the control formula and $2(7 \%)$ of the 29 patients who received the supplemented formula developed diarrhea 